\title{
Exons and Introns Characterization in Nucleic Acid Sequences by Time-Frequency Analysis
}

\author{
Umberto S.P. Melia, Francesc Clarià, Juan J. Gallardo, Pere Caminal, Alexandre Perera, and \\ Montserrat Vallverdú
}

\begin{abstract}
A current problem in deoxyribonucleic acid (DNA) sequence analysis is to determine the exact locations of the genes and also in eukaryotes, the protein-coding regions in the mRNA primary transcript (pre-mRNA).The conversion into discrete numerical values of the symbols associated to the nucleotides of these sequences allows for a signal to address the problems related to localization and annotation of genes. In this work, thermodynamic data of free energy changes $\left(\Delta G^{\circ}\right)$ on the formation of a duplex structure of DNA or RNA are used to convert the symbols into numerical values associated with the nucleotide sequence pre-mRNA. This study presents an analysis, based on techniques of timefrequency representation of a large number of gene sequences, in order to find variables related to pre-mRNA that could best characterize and discriminate coding regions from non-coding regions. It has been found that instantaneous frequency variables and instantaneous spectral energy variables in different frequency bands, allowed exons and introns to be correctly classified with more than $85 \%$.
\end{abstract}

\section{INTRODUCTION}

G ENOME contains all the instructions necessary to manufacture and keep alive an organism. Cell nucleus includes the deoxyribonucleic acid (DNA) composed of a succession of four different nucleotides: Adenine (A), Thymine (T), Cytosine (C), Guanine (G). Sequences of these nucleotides form long chains. In the case of the human genetic code these chains contain 3000 million of bases divided into 23 pairs of chromosomes. Proteins are functional units of living organism [1]. For their synthesis, portions of the genome containing the coding sequences of the DNA molecule, called genes, are translated into a molecule called ribonucleic acid (RNA). When a gene is expressed, the DNA double strand is opened during the time required for the formation of a single-stranded copy of the sequence of the gene from the RNA, called the primary transcript pre-mRNA or mRNA. Subsequently, this string is subjected to RNA editing during which the sections that are

Manuscript received April 20, 2010. This work was supported within the framework of the CICYT grant TEC2004-02274 from the Spanish Government. U.S. P. Melia, P. Caminal A. Perera and M. Vallverdú are with Dept. ESAII, Centre for Biomedical Engineering Research, Universitat Politècnica de Catalunya, CIBER of Bioengineering, Biomaterials and Nanomedicine (CIBER-BBN), Barcelona, Spain; email: \{umberto.melia, montserrat.vallverdu, pere.caminal \}@upc.edu. J.J. Gallardo is with CIBER-BBN, email: joan.josep.gallardo@upc.edu. F. Clarià is with Dept. IIE, Lleida University, Spain; email: claria@diei.udl.es. not going to be translated into protein (introns) are removed. Only the meaningful sections (exons) that can be translated into protein are spliced to form a continuous sequence. The final chain is a continuous sequence of coding region that can be translated into a protein. In complex organisms, the primary RNA transcript could be alternatively edited, so that the initial part of some introns can become coding sequence in response to specific biological signals. In complex organisms, two levels of molecular machinery are involved in splicing of pre-mRNA transcripts: the basal machinery and gene regulation system.

The basal machinery, which is found in all organisms whose genome contains introns, consists of five small nuclear RNA molecules (sRNA) [2]. These molecules, which are formed by few nucleotides, bind to certain proteins to form the "spliceosome" complex that is responsible of recognizing the sites where the intron starts and ends, cutting them, expelling them from the pre-mRNA transcript and then assembling the exons to form mRNA [3]. For each intron, four nucleotide sequences work as signals that report to the "spliceosome" where to cut: at the start of the intron or cut point 5', at the end or cut point 3', in the middle or area of branching, and polypyrimidine stretch [4]. Moreover, the gene regulation system controls the process of splicing and cutting by driving the basal machinery to these cut point [5-6].

Because in higher organisms, the ratio of lengths between exons and introns is very large (about $0.15 \mathrm{~kb}$ and $10 \mathrm{~kb}$, respectively, for humans), there are difficulties in extracting regular features of the structure, allowing the functional sequences (exons) to be predicted. Classically, it has been opted for two approaches for the automatic annotation of uncharacterized DNA sequences: on one hand, the search for homology with sequences annotated in other genomes from expression data and, on the other, based on extracting statistical patterns in the region of the gene, known as $a b$ initio prediction. However, from another perspective, the analysis in the frequency domain could be of great interest in differentiation between exons and introns. Fourier analysis is a basic tool in the processing of these sequences [7]; however, it can only reveal the overall frequency of stationary signals, losing the time dependence. With the time-frequency representation, it is possible to observe the evolution of the periodicity and 
frequency components along time, allowing non-stationary signals to be efficiently analyzed [8].

Moreover, the conversion into numerical values of the symbols associated with the nucleotide sequence of DNA permits to address the problems related to localization and annotation of genes by signal processing. It is worth mentioning that the time is associated with the position of nucleotides along the gene sequence. Thus, it can be considered, without losing generality, that the numerical representation of a nucleotide sequence is a signal sampled at intervals of 1 second and $0.5 \mathrm{~Hz}$ of bandwidth.

In the present work, an analysis based on techniques of time-frequency representation of a large number of gene sequences was performed in order to find which variables, related to pre-mRNA, can better discriminate coding regions from non-coding regions in a sequence.

\section{MATERIALS AND METHOdOLOGY}

\section{A. Analyzed Database}

In this work, 500 gene sequences were processed, taken from a chromosome with the corresponding notation of the positions of exons and introns. These sequences were taken from the public database Ensembl [9] and organized by software developed by our research group.

\section{B. Numerical Representation of Genomic Sequences}

The thermodynamic values of enthalpy $\left(\Delta \mathrm{H}^{\circ}\right)$, entropy $\left(\Delta \mathrm{S}^{\circ}\right)$ and free energy $\left(\Delta \mathrm{G}^{\circ}\right)$ changes for the formation of a duplex structure of DNA or RNA can be calculated from the thermodynamic data library, based on the nearestneighbor interactions [10,11], with 10 combinations of base pairs of Watson-Crick: AA / TT, AT / TA, TA / AT, CG / $\mathrm{GC}, \mathrm{GC} / \mathrm{CG}, \mathrm{CT} / \mathrm{GA}, \mathrm{GA} / \mathrm{TC}, \mathrm{GT} / \mathrm{AC}, \mathrm{AC} / \mathrm{GT}, \mathrm{GG} /$ CC.

In this work, the Gibbs free energy $\left(\Delta \mathrm{G}^{\circ}\right)$ was used in order to convert into numeric values the symbols associated with the nucleotide sequence of pre-mRNA. The numbering sequence thus obtained is a sequence of symbols with the particularity of providing a physical meaning. It accounts for the ease with which a number of nucleotide binds to its complementary set, a circumstance that occurs continuously during the editing process in the pre-mRNA sequence. That happens, during the cutting of introns and the union of exons, in order to generate the mRNA sequence that carries the protein code.The obtained sequences are a succession of thermodynamic values that can be assimilated to a non-stationary stochastic process with a non-zero mean value.

\section{Time-Frequency Representation Tools}

For each gene, Choi-Williams distribution (CWD) [12] was applied to numerical sequences, after the calculation of the analytical signal, by using the expression (1) with the Wigner distribution (WD) (2) and the Choi-Williams (CW) exponential (3).

$$
\begin{gathered}
T F R_{x x}(t, f)=\iint \Psi\left(t-t^{\prime}, f-f^{\prime}\right) W_{x x}\left(t^{\prime}, f^{\prime}\right) d t^{\prime} d f^{\prime} \\
W_{x x}(t, f)=\int x\left(t+\frac{\tau}{2}\right) x^{*}\left(t-\frac{\tau}{2}\right) e^{-j 2 \pi f \tau} d \tau
\end{gathered}
$$

The formula (2) is the WD of a $x(t)$ signal, where $x *(t)$ is the complex conjugate signal of $x(t)$. The CWD (1) is obtained by the convolution between WD (2) and CW function (3). Finally, CWD function [12] is defined in timefrequency domain.

$$
\Psi(t, f)=\sqrt{\frac{4 \pi}{\sigma_{c}}} e^{-4 \pi^{2} \frac{(t f)^{2}}{\sigma_{c}}}
$$

The function (3) preserves the properties of the WD [13], such as the marginal properties and instantaneous frequency. Besides that, it is capable of reducing WD interference thanks to an adequate choice of $\sigma_{c}$ parameter. In this case, $\sigma_{c}=0.05$ represents an efficient value.

In order to calculate variables that might reflect characteristics of the signal in different frequency ranges, the spectrum was divided into the following frequency bands: VLF, 0-0.1 Hz; LF, 0.1-0.2 Hz; HF, 0.2-0.4 Hz; VHF, 0.4-0.5 Hz; TF, 0-0.5Hz.

Instantaneous spectral energy was calculated for each gene signal as the CWD integral in frequency in each of the bands. Subsequently, for each band, the instantaneous frequency function was calculated [13] as the average frequency of the spectrum along the time, using equation (4).

$$
f_{s}(t)=\frac{\sum_{n=1}^{N} f_{n} \cdot T F R_{x x}\left(t, f_{n}\right)}{\sum_{n=1}^{N} T F R_{x x}\left(t, f_{n}\right)}
$$

where $N$ is the number of samples in frequency axis.

Figure 1 shows an example of CWD normalized by the maximum value of a gene (named AGT), with exons and introns differentiated by lines.

\section{Shannon and Rényi Entropies}

In order to measure the complexity of the time-frequency signal response, Shannon entropy, equation (5), and Rényi entropy, equation (6), were applied to each sequence of instantaneous frequency and instantaneous spectral energy calculated from each CWD. Also, the same techniques were applied to the original sequences of the Gibbs energy for each gene. In equation (6), the large probabilities most influence the Rényi entropy when $q>1$ and the small probabilities most influence the value of Rényi entropy when $0<q<1$. The Rényi entropy converges to the Shannon entropy when $q \rightarrow 1$. 


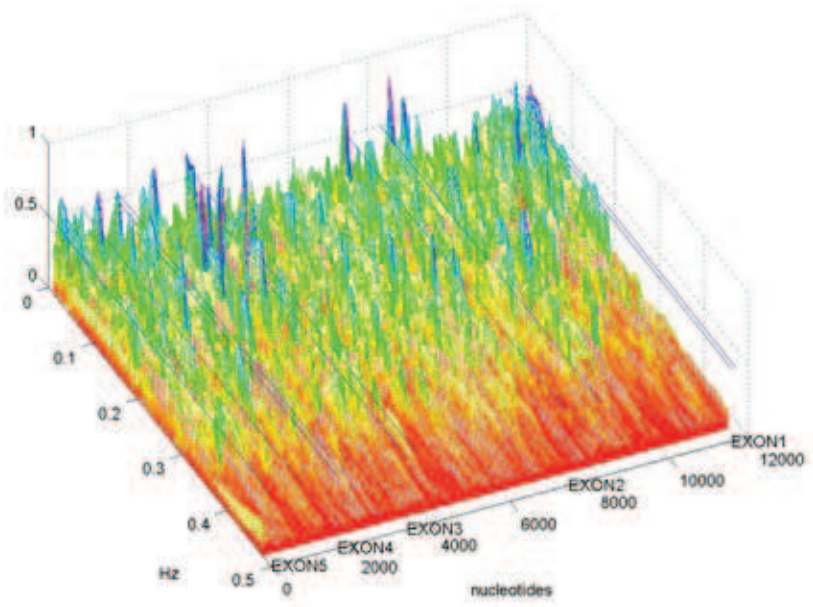

Fig. 1. CWD of AGT gene, blue lines divide exon from intron regions.

$$
\begin{aligned}
& \text { ShaEn }=-\sum_{i=1}^{Q} P(i) \cdot \log _{2}(P(i)) \\
& \operatorname{Re}(q)=\frac{1}{1-q} \log _{2}\left(\sum_{i=1}^{Q} P^{q}(i)\right)
\end{aligned}
$$

where $Q=8$ represents the amount of levels in which the signals are quantized. Values of Rényi entropies for $q=\{0.15,0.25,0.5,2,3\}$ were calculated.

\section{E. Definition of Variables}

Several variables were defined for instantaneous frequency, instantaneous spectral energy and Gibbs energy along nucleotide position. For each signal, the following variables were calculated: mean, standard deviation, maximum and minimum value, the position of the maximum and minimum value, Shannon and Rènyi entropies. All these variables were obtained from the complete sequence and from the $n=\{25,50,75,100\}$ nucleotides at the start and at the end of each intron and exon. However, Shannon and Rènyi entropies were calculated only for the complete sequence in order to avoid uncertainty in the calculation of the probability of short signals. Instead, the nucleotide position of the peak was calculated only for the $n$ samples at the start and at the end of intron and exon sequences. Since this value depends of the length of the sequence, this could influence the statistical classification. In these $n$ samples, it is considered that there is a peak if this peak value exceeds $75 \%$ of the maximum value of the entire sequence; otherwise it is assumed that no maximum value exists in these regions.

\section{F. Statistical Analysis}

The Mann-Whitney test, a non-parametric test and therefore independent from the type of distribution, was used for the statistical analysis in order to compare data from two independent populations and to assess whether these two populations come from the same distribution. The considered populations were: exons and introns, labeled as group 1 and group 2, respectively.

For each variable, the statistical significance level $p$ of the Mann-Whitney test was calculated and the classification was made with a quadratic function (7). In this formula, the subscript $i$ denotes the respective class or group; the subscripts $j=1,2, \ldots, m$ denote the variable under consideration taken from the total amount of calculated variables; $c_{i}$ is a constant for each class, $w_{i j}$ is the weight for the $j$-th variable in the computation of the classification score for the $i$-th class; $x_{j}$ is the observed value for the respective $j$-th variable. The resultant classification score is $S_{i}$.

$$
S_{i}=\left(c_{i}+w_{i 1} x_{1}+w_{i 2} x_{2}+\ldots+w_{i m} x_{m}\right)^{2}
$$

For classification, the first step was to build a discriminant function (7) by calculating the coefficients $w_{i}$. The aim is making the $S_{i}$ value different as much as possible between the classes (introns and exons) trying to maximize the equation (8).

$$
R=\frac{\text { quadratic sum of differences between classes }}{\text { quadratic sum of differences into classes }}
$$

The classification algorithm uses half of the number of genes for training, in order to build a classification function, and the other half for validating its accuracy. The percentage of correctly classified exons and introns was respectively calculated as the rate between number of exons or introns correctly classified and total number of exons or introns, respectively.

On the other hand, the use of a genetic algorithm (GA) gives advantages for obtaining a set of variables, chosen from the total amount, so that they provide high accuracy in classification of exons and introns by the calculation of (7). This algorithm performs a number of iterations in an attempt to get good percentages with different variables randomly chosen. In this way, it can be done a more thorough analysis of the total combinations of all defined variables.

\section{RESULTS AND DISCUSSION}

Only instantaneous frequency entropies were able to characterize $(p<0.0005)$ exons and introns with a percentage higher than $60 \%$ of well classified sequences. Table I shows the mean value, the standard deviation and the percentages of correctly classified exons and introns for the best variables: Rènyi entropy $(q=0.5)$ in VHF band (VHF_RE05), Shannon entropy in HF and LF band (HF_ShaEn and LF ShaEn) calculated in instantaneous 
frequency signals. It can be noted that the mean value of entropy of exons is always higher than the entropy of the introns.

TABLE I

BEST VARIABLES TAKEN SINGULARLY

\begin{tabular}{lllll}
\hline \hline Variable & $\begin{array}{l}\text { Exon (E) } \\
\mathrm{m} \pm \sigma\end{array}$ & $\begin{array}{l}\text { Intron (I) } \\
\mathrm{m} \pm \sigma\end{array}$ & $\%$ E \% I \\
\hline \hline VHF_RE05 & $2.7548 \pm 0.1547$ & $2.6265 \pm 0.1588$ & 67.9 & 66.0 \\
HF_ShaEn & $2.9351 \pm 0.1231$ & $2.8186 \pm 0.1395$ & 73.0 & 62.6 \\
LF_ShaEn & $2.9289 \pm 0.1214$ & $2.8158 \pm 0.1372$ & 71.3 & 62.7 \\
\hline
\end{tabular}

Variables of instantaneous frequency entropy of complete sequence: $\mathrm{m}$, mean value; $\sigma$ standard deviation; statistical significance level $\mathrm{p}<0.0005$, comparing $\mathrm{E}$ and I.

Combining variables by GA have permitted to obtain higher percentages of correctly classified exon and intron sequences. The best combination was found with variables related to instantaneous spectral energy and instantaneous frequency of the complete sequence of exon versus the 100 nucleotides at the start (AEIS) and at the end (AEIE) of intron. In Table II, the best variables for both cases are shown with the percentages of correctly classified exons and introns.

TABLE II

BEST COMBINATION OF VARIABLES

\begin{tabular}{ll}
\hline \hline AEIS & AEIE \\
\hline \hline Inst_Freq_mean & Inst_Freq_mean \\
Inst_Freq_std & Inst_Freq_std \\
Inst_Freq_VLF_mean & Inst_Freq_VLF_mean \\
Inst_Freq_LF_mean & Inst_Freq_HF_mean \\
Inst_Freq_HF_mean & Inst_Freq_VHF_mean \\
Inst_Freq_VLF_std & Inst_Freq_VLF_std \\
Inst_Freq_LF_std & Inst_Freq_LF_std \\
Inst_Freq_HF_std & Inst_Freq_HF_std \\
Inst_Freq_VHF_std & Inst_Sp_En_LF_mean \\
Inst_Sp_En_VLF_std & Inst_Sp_En_VLF_std \\
Inst_Sp_En_HF_std & Inst_Sp_En_HF_std \\
\hline $\mathrm{E}=84.9 \%$ & $\mathrm{E}=86.5 \%$ \\
$\mathrm{I}=84.8 \%$ & $\mathrm{I}=86.5 \%$ \\
\hline \hline & Percentages of exons $(\mathrm{E})$ and introns $(\mathrm{I})$ correctly classified after \\
combination of variables. Inst: instantaneous; Freq: frequency; $S p:$ & spectral; En: energy; std: standard deviation. \\
\hline
\end{tabular}

\section{CONCLUSIONS}

The start and end part of introns (cut point 5' and 3', respectively) and the polypyrimidine tract, that is also toward the end of intron, contain nucleotide sequences that work as signals for indicating where the spliceosome have to cut. For this, the signals obtained in this region, with the conversion of nucleotides in numerical signals, must be of deterministic type compared with the signals obtained from exons that do not need to notify to the spliceosome about their position. Exonic splicing enhancers and silencers are exception. This hypothesis is supported by the fact that the mean value of the entropy of the sequences of instantaneous frequency and instantaneous spectral energy is always higher in exons than in introns, indicating that introns are structurally less complex, more regular. It is noteworthy that variables related to Gibbs energy sequences are not sufficient to correctly classify exons from introns because they percentages of accuracy are lower than the ones of CWD variables.

This work represents a preliminary study about the advantages taken from the application of time-frequency representation on the characterization of nucleic acid sequences. Additional tests will be made in order to validate the results using more data taken from other chromosomes.

\section{ACKNOWLEDGMENT}

CIBER of Bioengineering, Biomaterials and Nanomedicine is an initiative of ISCIII.

\section{REFERENCES}

[1] International Human Genome Sequencing Consortium. Initial sequencing and analysis of the human genome. Nature, vol. 409, 2001, pp. 860-921.

[2] J. Valcárcel R.K Gaur, R. Singh and M.R. Green, "Interaction of U2AF65 RS region with pre-mRNA branch point and promotion of base pairing with U2 snRNA," Science, vol. 273, n. 5282, 1996, pp. 1706-1709.

[3] P. Zuo and T. Maniatis, "The splicing factor U2AF35 mediates critical protein-protein interactions in constitutive and enhancer dependent splicing," Genes Development, vol. 10, 1996, pp. 13561368 .

[4] B.R. Graveley, K.J. Hertel and T. Maniatis, "The role of U2AF35 and U2AF65 in enhancer-dependent splicing," $R N A$, vol. 7, 2001, pp. 806-818.

[5] B.J. Blencowe, "Exonic splicing enhancers: Mechanism of action, diversity and role in human genetic disease," Trends Biochem Sci, n. 25, 2000, pp. 106-110.

[6] J.L. Kan and M.R Green, "Pre-mRNA splicing of IgM exons M1 and M2 is directed by a juxtaposed splicing enhancer and inhibitor," Genes Development, vol. 13, 1999, pp. 462-471.

[7] M. Akhtar, J. Epps, and E. Ambikairajah, "Signal processing in sequence analysis: advances in eukaryotic gene prediction," IEEE Journal of Selected Topics in Signal Processing, vol. 2, n. 3, 2008, pp. 310-321.

[8] J. Ning, C.N. Moore, and J.C. Nelson, "Preliminary wavelet analysis of genomic sequences," IEEE Computer Society Bioinformatics Conference (CSB'03), 2003, pp. 509.

[9] Ensembl release 57, 2010. Available: http://www.ensembl.org

[10] K.J. Breslauer, R. Franks, H. Blockers, and L.A. Marky, "Predicting DNA duplex stability from the base sequence," Biochemistry. Proc. Natl. Acad. Sci. USA, vol. 83, 1986, pp. 3746-3750.

[11] N. Sugimoto, S. Nakano, M. Yoneyama, and K. Honda, "Improved thermodynamic parameters and helix initiation factor to predict stability of ADN duplexes," Oxford University Press. Nucleic Acids Research, vol. 24, n. 22, 1996, pp. 4501-4505.

[12] F. Hlawatsch and G.F. Boudreaux-Bartels, "Linear and quadratic time-frequency signal representations," IEEE SP-Magazine, 1992, pp. 21-67.

[13] L. Cohen, "Time-frequency analysis," Prentice Hall Signal Processing Series, 1995. 